\title{
The future of hybrid imaging-part 3: PET/MR, small-animal imaging and beyond
}

\author{
Thomas Beyer • Lutz S. Freudenberg • \\ Johannes Czernin • David W. Townsend
}

Received: 1 October 2010 /Revised: 4 January 2011 /Accepted: 18 February 2011 /Published online: 25 March 2011

(C) The Author(s) 2011. This article is published with open access at Springerlink.com

\begin{abstract}
Since the 1990s, hybrid imaging by means of software and hardware image fusion alike allows the intrinsic combination of functional and anatomical image information. This review summarises in three parts the state of the art of dual-technique imaging with a focus on clinical applications. We will attempt to highlight selected areas of potential improvement of combined imaging technologies and new applications. In this third part, we discuss briefly the origins of combined positron emission tomography (PET)/magnetic resonance imaging (MRI). Unlike PET/ computed tomography (CT), PET/MRI started out from developments in small-animal imaging technology, and, therefore, we add a section on advances in dual- and multimodality imaging technology for small animals. Finally, we
\end{abstract}

T. Beyer $(\bowtie)$

cmi-experts $\mathrm{GmbH}$,

Pestalozzistr 3,

8032 Zürich, Switzerland

e-mail: thomas.beyer@cmi-experts.com

T. Beyer $\cdot$ L. S. Freudenberg

Department of Nuclear Medicine, University Hospital Essen,

Essen, Germany

L. S. Freudenberg

Department of Nuclear Medicine, ZRN,

Grevenbroich, Germany

J. Czernin

Department of Molecular and Medical Pharmacology,

David Geffen School of Medicine, UCLA,

Los Angeles, CA, USA

D. W. Townsend

Singapore Bioimaging Consortium,

11 Biopolis Way, 02-02 Helios,

Singapore 138667, Singapore highlight a number of important aspects beyond technology that should be addressed for a sustained future of hybrid imaging. In short, we predict that, within 10 years, we may see all existing multi-modality imaging systems in clinical routine, including PET/MRI. Despite the current lack of clinical evidence, integrated PET/MRI may become particularly important and clinically useful in improved therapy planning for neurodegenerative diseases and subsequent response assessment, as well as in complementary locoregional oncology imaging. Although desirable, other combinations of imaging systems, such as single-photon emission computed tomography (SPECT)/MRI may be anticipated, but will first need to go through the process of viable clinical prototyping. In the interim, a combination of PET and ultrasound may become available. As exciting as these new possible triple-technique - imaging systems sound, we need to be aware that they have to be technologically feasible, applicable in clinical routine and cost-effective.

Keywords Hybrid imaging · PET · MRI P PET/MR ·

Small-animal imaging

"Prediction is very difficult, especially if it is about the future." Niels Bohr (1885-1962)

\section{Positron emission tomography (PET)/magnetic resonance imaging (MRI)}

Background and reasoning

In view of the success of PET/CT, the expectations for any new combination, such as PET/MR, are very high. MRI is a more versatile imaging technique than $\mathrm{CT}$ in that it measures a 
number of physiological and metabolic characteristics of human tissue [1]. MRI goes beyond plain anatomical imaging by offering a multitude of endogenous contrast agents and high capability in differentiating soft tissues, as well as many exogenous contrast media ranging from gadolinium-based agents to highly specific cellular markers.

Magnetic resonance spectroscopy (MRS), for example, can be used to dissect the molecular composition of tissues by applying selective radiofrequency excitation pulses. Functional processes in living subjects can also be studied by diffusion-weighted (DW) MRI. Here, the magnetic field, generated by different gradients, is used to map phase differences in the MRI signal that are caused by diffusing molecules. DW-MRI has potential clinical applications ranging from diagnosing ischaemia, cancer, multiple sclerosis, or Alzheimer's disease to general fibre tracking via diffusion tensor imaging (DTI), and it is not restricted to the brain. In addition, functional MRI (fMRI) studies can be performed during the same examination. Functional MRI studies are frequently based on the BOLD (blood oxygen level-dependent) effect. This effect describes the fact that the magnetic properties of oxygenated and deoxygenated haemoglobin in the blood are different and, therefore, produce different signals when imaged with $\mathrm{T} 2 *$-sensitive MRI sequences. The BOLD effect also has certain applications in cancer imaging, such as to study tumour angiogenesis, tumour oxygenation and brain activation in relevant areas before surgical resection.

Lately, MRI has become a whole-body imaging technique as a consequence of the introduction of parallel imaging techniques. Image acquisition times have been shortened, thus allowing whole-body MRI examinations with high spatial resolution in less than $1 \mathrm{~h}$. Initial results show that whole-body MRI is a promising technique in oncology, especially for the detection of metastases and haematological malignancies.

In summary, MRI holds great potential for replacing CT as the complementary technique to PET in dual-technique tomographs and in selected indications where MRI outperforms CT already. In theory, MRI seems a perfect anatomical complement to PET.

Technical challenges, concepts and methodological aspects

The development of combined PET/MRI systems started in the late 1990's. Given the design of standard PET [and single-photon emission computed tomography (SPECT)] detectors based on photomultiplier tubes (PMTs), a PET/ MRI configuration is obviously technically more challenging than the combination of PET (or SPECT) and CT because phototubes are sensitive even to low magnetic fields (Fig. 1). MRI demands very high field homogeneity, and the presence of PET detectors within this field could interfere with the MRI. Conversely, the PET detectors have to withstand not only a high static field level (up to $3 \mathrm{~T}$ for clinical MRI), but also the rapidly changing field gradients required by the imaging process.

Hammer and co-workers were one of the first groups to address some of these issues in the mid-1990s. They proposed to place the PET scintillator blocks inside a clinical MRI and to extract the information from the scintillator through light guides that are fed into detector electronics situated outside the primary magnetic field of the MRI system [2, 3]. In the mid 1990s, Shao and co-workers developed a small ring of PET detectors $3.8 \mathrm{~cm}$ in diameter for pre-clinical, small animal imaging [4]. Although subsequent prototypes were suggested (e.g. Slates et al. [5], Pichler et al. [6] and Judenhofer et al. [7]), PET/ MRI was destined to remain in the pre-clinical arena for another decade [8] until, in 2006, the first simultaneous MR and PET images of the human brain were acquired [9].

Figure 2 shows existing hardware concepts for clinical PET/MRI. In essence three approaches exist towards PET/ MRI: separate gantries operated in different rooms (a), gantries arranged in line with the main scanner axis with a patient handling system mounted in between (b) and a fully integrated system (c). The third design, presented in 2006, and also the most challenging (Fig. 2c), is based on a PET detector ring designed as an insert that can be placed inside a Siemens 3-T Trio MR system (Siemens Healthcare). This prototype system (BrainPET) was intended for brain imaging only. The PET insert has an internal diameter of $35.5 \mathrm{~cm}$ and comprises 192 LSO (lutetium oxyorthosilicate) detector blocks arranged in six rings. Each LSO block comprises a $12 \times 12$ matrix of $2.5 \times 2.5 \times 20 \mathrm{~mm}^{3}$ crystals for an axial field of view (FOV) of $19.25 \mathrm{~cm}$ [9]. Each detector block is directly coupled to a compact $3 \times 3$ APD (avalanche photo diode) array. The point source sensitivity of the PET system measured with a line source in air is $5.6 \%$ and the spatial resolution is $2.1 \mathrm{~mm}$ at the centre of the FOV. No degradation of the MR images was observed due to the presence of the PET detectors and no detrimental effect on the performance of the PET detectors was observed for a number of standard MR pulse sequences [9, 32].

The co-planar PET/MRI concept (Fig. 2b), first presented in 2010, is based on a tandem design of a whole-body timeof-flight (TOF) PET system and a 3-T Philips Achieva MR system (Philips Healthcare, Cleveland, USA) with a rotating table platform in between. Through minor modifications of the PET detector system (e.g. orientation of the PMT, minor shielding) the PET gantry can be operated in close proximity to the 3-T MRI system.

The first design was proposed by GE Healthcare in late 2010 and is so far available as prototype technology only. This design is based on a combination of a dual-technique PET/CT and a 3-T MRI system, which are operated in separate, adjacent rooms; patients are shuttled from one 
Fig. 1 a Example of photomultiplier tubes (PMT)-bismuth germanate (BGO) block detector from a clinical PET system. Readout is performed using the PMTs that are connected to the pixellated scintillator block. Light sharing is used to distribute light originating from a single pixel between the readout PMTs (P1-P4). The position of the incident annihilation photon event can be calculated using an Anger-weighting of the measured signals (b). b Schematics of the detection process from annihilation to stopping the annihilation of photons in the crystal and signal transformation inside the PMT. c Conventional PET detectors (see a) work only outside magnetic fields $(\mathrm{B}=0)$. If a PMT is operated inside a magnetic field $(\mathrm{B}>0)$, then the multiplier step is distorted and the readout map severely distorted. $\mathbf{d}$ Avalanche photodiode (APD)based detectors are semiconductors that can be operated in magnetic fields, even at higher field strengths. Images courtesy Prof. B. Pichler, Tübingen

\section{(A)}
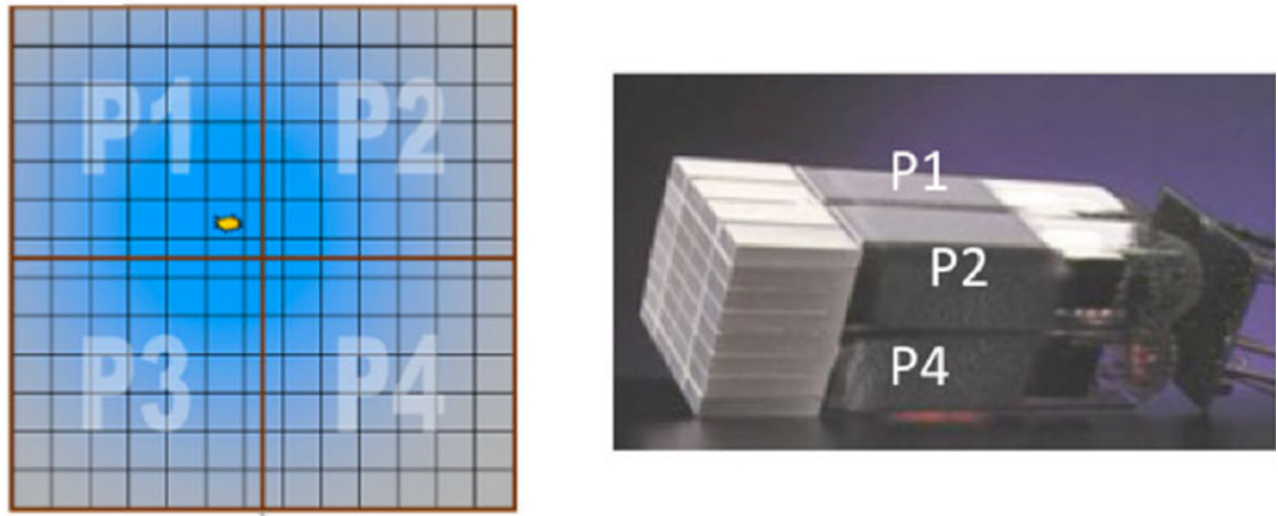

(B)

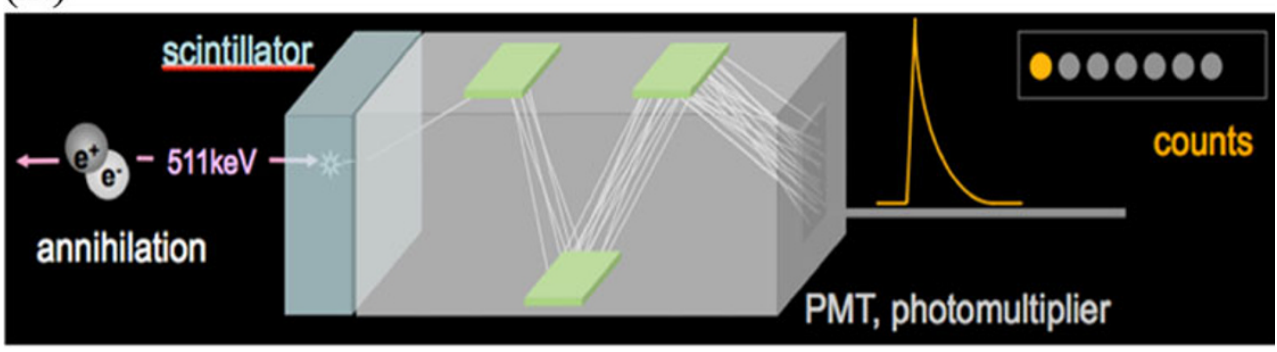

(C)

Conventional PET detectors
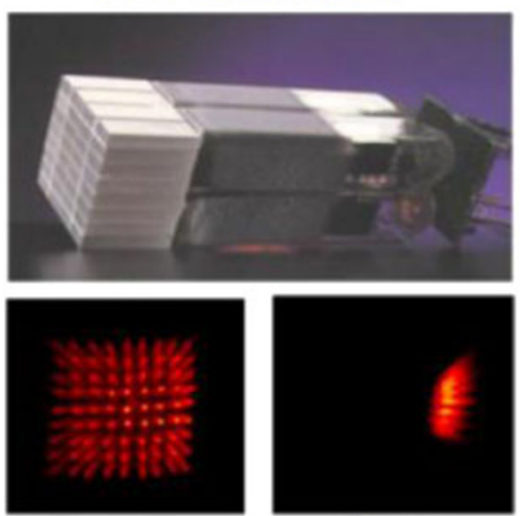

$B=0$

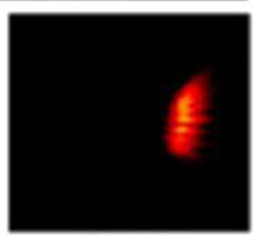

$B \neq 0$

(D)

\section{APD-based PET detectors}
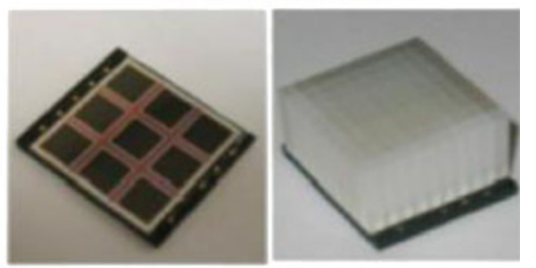

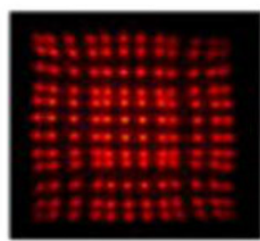

$B=0$

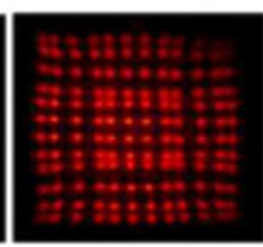

$B \neq 0$ system to the other without getting off the bed. This approach substitutes the challenges of hardware integration for considerable logistical challenges in timing access to the two systems while minimising patient motion in between examinations. However, this approach has been argued as the most cost-effective compared with fully integrated PET/MRI, based on workflow aspects and machine utilisation [10].

In an extension to the integrated design concept of Fig. 2c, a similar system was proposed in late 2010 that merged a whole-body PET with a 3-T MRI system to allow for simultaneous whole-body imaging. Just like the
BrainPET PET/MR prototype, this system is based on LSO-APD PET detector technology, which is integrated into the MR gradient coil system offering a 60- cm gantry opening (versus a $35-\mathrm{cm}$ gantry opening for the brain prototype).

In addition to the technical challenges of combining PET and MRI, which increase with the amount of PET-MRI system integration, the necessary attenuation correction factors (ACFs) for the PET emission data must be derived from the PET/MRI measurements [11]. While in PET/CT PET attenuation data can be derived from transforming available CT transmission images into maps of attenuation 


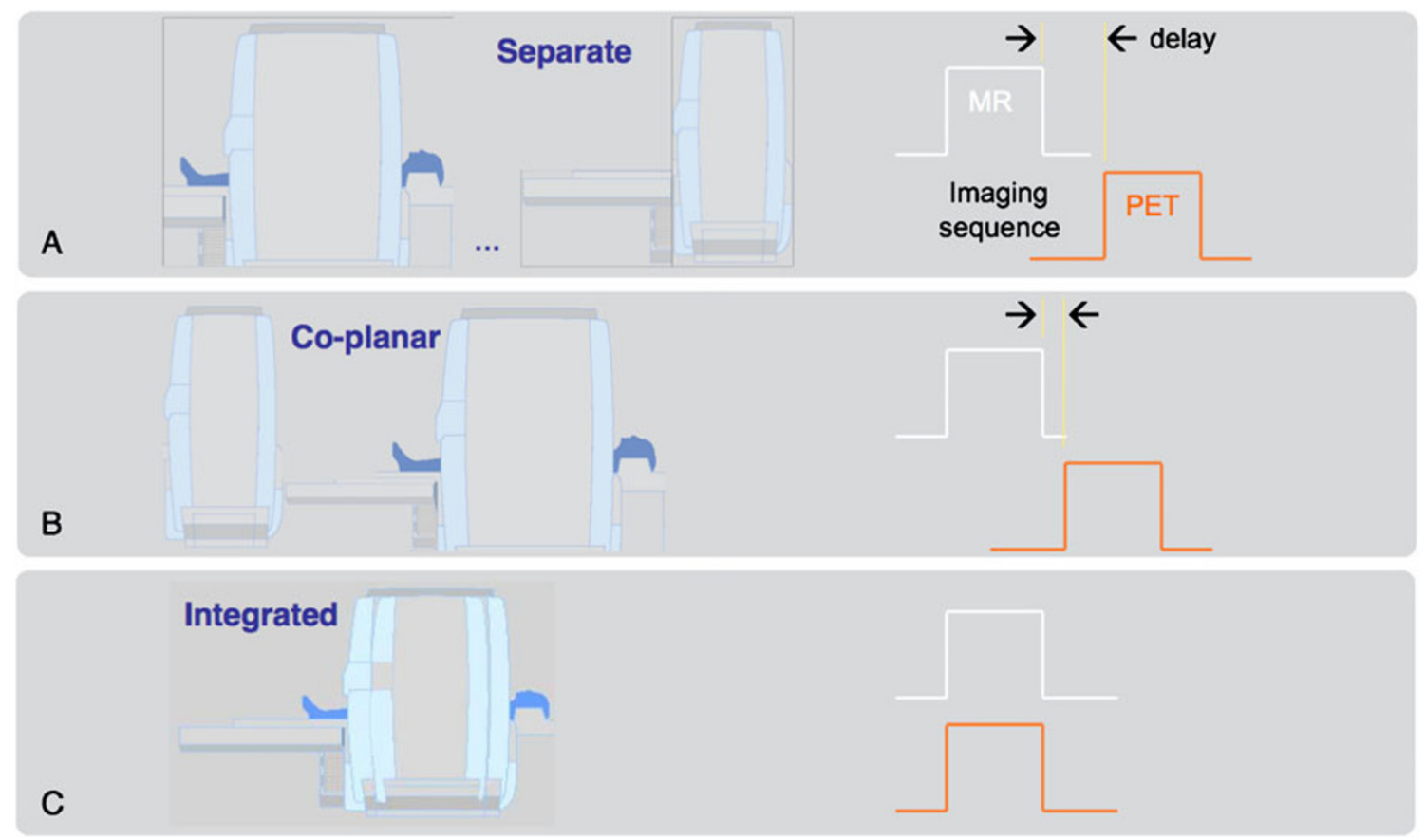

Fig. 2 Different designs for combined clinical PET/MR systems: (A) patients can be shuttled between separate MR and PET(/CT) systems operated in different rooms, (B) patients are positioned on a common table platform between stationary PET and MR systems; the delay between the MR- and PET-examination is reduced (Philips Healthcare), and (C) patients are positioned inside an integrated PET/MR gantry (Siemens Healthcare) with a PET insert that is mounted within a whole-body MR offering simultaneous PET/MR acquisitions coefficients at $511 \mathrm{keV}$, no such transmission data are available for PET/MRI. This is primarily due to the lack of physical space to host a transmission source. Second, a rotating metal-encased transmission source, whether X-ray tube, rod or point sources would lead to grave cross-talk effects with the MR magnetic field. And finally, the available MR images represent, in essence, proton densities that cannot be directly translated into maps of electron densities as obtained from $\mathrm{CT}$ transmission measurements. For example, air and cortical bone yield no significantly measurable MR signal, whereas the difference in their photon attenuation properties is 2,500 HU on CT images (Fig. 3). Therefore, PET/MRI requires novel approaches to MR-based attenuation correction (MR-AC).

Originally, segmentation-based approaches have been proposed to classify tissues on MR images and to assign respective attenuation coefficients. This approach seems to work well in brain imaging [12]. However, MR-based attenuation correction (MR-AC) in extra-cerebral applications is much more demanding [13]. Therefore, atlas-based approaches have been suggested [14] and torso data [15].

The principle of the atlas approach is to align the MRI acquired for the PET/MRI study with an average MR image from an atlas comprising pairs of registered MR and CT data sets. The same transformation determined from the alignment of the MRI of the patient with the MRI in the atlas can be applied to the CT volume from the atlas. A combination of the registered CT image volume and the patient-specific MRI can be used to generate a pseudo-CT map of the PET/MRI study from which the ACFs can be derived [16]. In view of the absence of an MR bone signal, the bone structures can be extracted from the registered atlas $\mathrm{CT}$ and combined with an MR image segmented for air and soft tissue.

Combined PET/CT has been clinically very successful and may well serve as a benchmark for the development of PET/MRI. However, despite the success and wide distribution of PET/CT, there are some shortcomings in the use of $\mathrm{CT}$ as the anatomical complement to PET. CT uses a source of ionising radiation for imaging and, therefore, adds significant radiation dose to the overall examination [17], which may raise concerns in selected populations like adolescents and women [18]. Further, CT provides comparatively low soft-tissue contrast, which is exacerbated when CT contrast material is being used. MRI, on the other hand, does not suffer from these two major disadvantages and, in addition, offers more advanced functional imaging information, such as DWI or MRS, without adding to the overall radiation exposure burden. Other safety concerns do, however, apply to MRI and PET/MRI as discussed by Brix et al. [19] and mandate the close observation of local heat tolerance effects in response to specific absorption rates (SAR) from radiofrequency (RF) exposure and careful pre-examination patient interviews on the presence or 


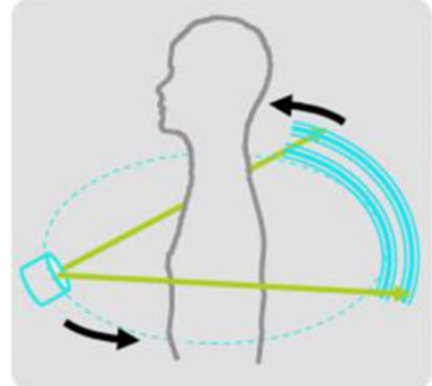

CT $\rightarrow$ electron density
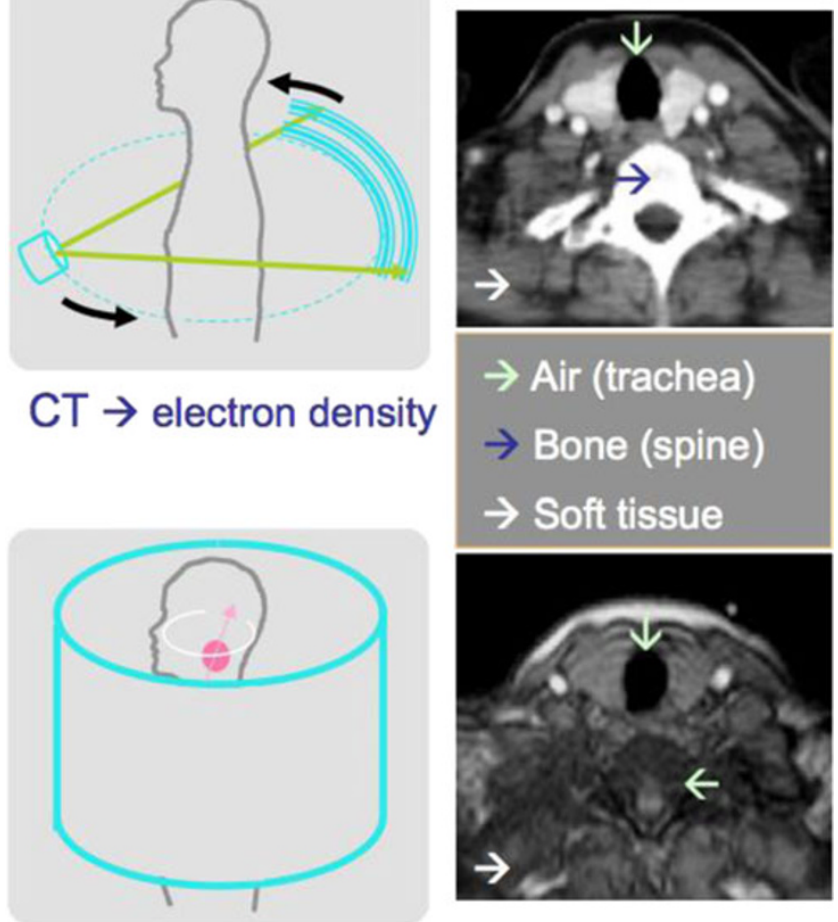

$\rightarrow$ Soft tissue

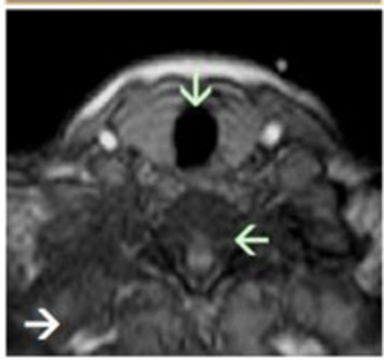

\section{$\mathrm{MR} \rightarrow$ proton density}

Fig. 3 MR-based attenuation correction is demanding as the appearance of air (turquoise arrow) and bone (blue arrow) on MR images is very similar despite their significantly different attenuation coefficients for ionising radiation (see $\mathrm{CT}$, top) absence of passive implants, which may interfere with the MRI protocol, or disqualify the patient from this examination all together.

\section{Clinical expectations for PET/MRI}

The combination of PET and MRI in a single imaging system has the potential to become the ultimate multi-modality imaging technology, combining anatomical, functional, metabolic and multi-parametric imaging. Nonetheless, it is difficult to propose clinical applications of combined PET/ MRI at this stage, where first prototype systems are being validated in clinical and research settings [20]. Given the fact that numerous studies exist on the use of retrospectively aligned PET and MRI (as well as SPECT and MRI), it is fair to say that hardware-fusion PET/MRI has the potential to dominate over standalone imaging in certain areas of noninvasive imaging [21].

PET/MR in neurology The potential areas of application of combined PET/MRI extend far beyond high-contrast image fusion. Brain studies, for example, benefit greatly from the additional morphological information provided by MRI (Fig. 4). Combined amino acid PET and MRI is likely to enhance the diagnostic sensitivity for gliomas and may allow a closer correlation between the tracer uptake and the metabolic changes (e.g. choline peaks in MR spectroscopy) in the neoplastic tissue [22]. Likewise, arterial spin-
Fig. 4 a Patient with meningioma in the right frontal lobe. Axial MR and simultaneous PET/MR images through the lesion: T2-weighted MRI, ${ }^{68} \mathrm{Ga}$-DOTATOC PET. b A 42-year-old man with a neurocytoma. PET/MR images were acquired simultaneously following injections of

${ }^{11} \mathrm{C}$-methionine (left). Simultaneously acquired chemical shift imaging MRS provides a map of the choline to $N$-acetyl-aspartate ratio (centre). Simultaneous diffusion tensor imaging (DTI) shows the clear relationship with the adjacent optic radiation. Cases courtesy of Drs. Boss, Bisdas and Schwenzer (UH Tübingen, Department of Radiology)

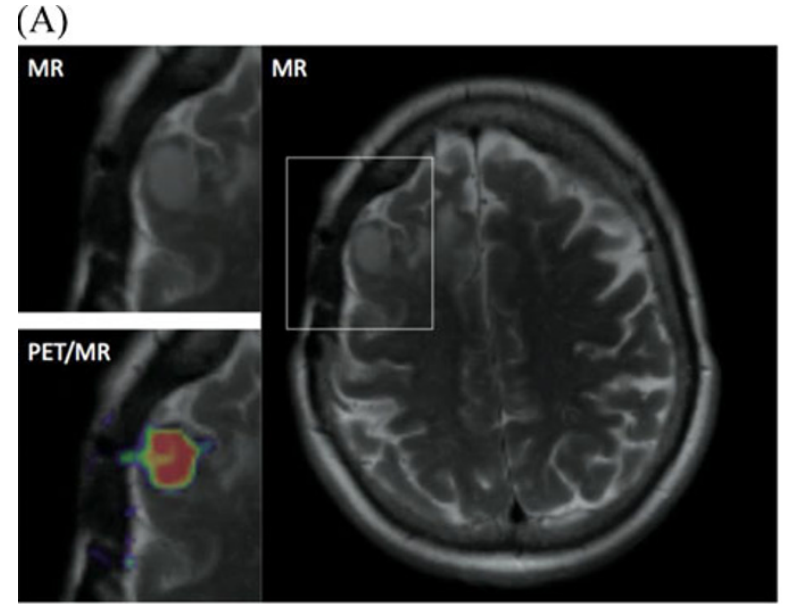

(B)

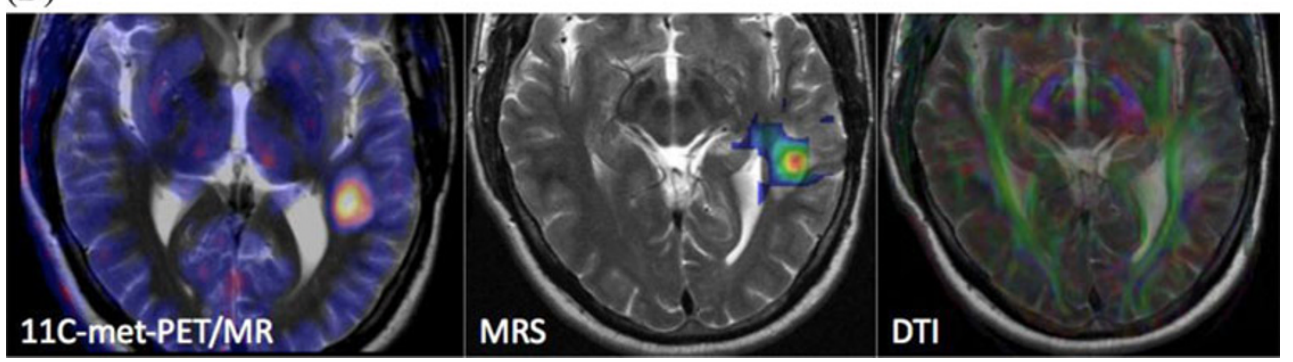


labelling estimations of perfusion and diffusion changes occurring in low-grade gliomas may be studied in conjunction with each PET-tracer image to establish reliable disease markers. Consequently, the "wait-and-see" approach to low-grade gliomas may be optimised with regard to the timing and extent of surgery. For the diagnosis of degenerative and neoplastic diseases, DWI-MRI helps overcome the shortcomings of morphology imaging only. Contrast-enhanced dynamic MRI, which may play a more decisive role for therapy outcome in the future, may now be compared with the PET-tracer kinetics as the "gold standard". Boss and co-workers recently evaluated simultaneous PET/MRI for assessing intracranial tumours using ${ }^{11} \mathrm{C}$-methionine or ${ }^{68} \mathrm{Ga}$-DOTATOC (Fig. 4a). They demonstrated image quality and quantitative data achieved from PET/MRI to be similar to that using PET/CT [23]. While several of the above aspects await further clinical testing, integrated PET/MRI appears to have great potential in neuroscience research (Fig. 4b), particularly for multiparametric analysis of complex functions in neural networks, for the imaging of complex molecular processes of gene transfer and cell transplantation and for translational research from pre-clinical into clinical use [24].

PET/MRI in oncology: PET/MRI may be useful for extracerebral oncology applications, but a key application has yet to be found. In an early study from 2003, Antoch and co-workers compared whole-body FDG-PET/CT and multistation MRI in a heterogeneous group of cancer patients and concluded that FDG-PET/CT performed better in overall TNM staging than MRI and, therefore, should be recommended as a possible first-line technique for wholebody tumour staging [25]. In a recent review, Antoch and Bockisch summarised key studies from the literature and their own experience [26] and conclude that PET/MRI may be expected to be more accurate than PET/CT for T-staging in all indications in which MRI is more accurate than CT, while similar accuracies are to be expected for N-staging. For M-staging, potential advantages of PET/MRI will depend on the site of the metastases. Other extra-cerebral applications of PET/MRI are currently being assessed, but no real hypothesis can yet be made with regard to the future clinical potential.

One of the primary strengths of MRI is its ability to provide anatomical detail in addition to detecting abnormalities within bony structures (e.g. marrow, joint spaces). $\left[{ }^{18} \mathrm{~F}\right]$-FDG PET is useful in the diagnosis of acute infections and is an accurate imaging technique to exclude the diagnosis of osteomyelitis. When combined and clinically available, PET/MRI may provide a more accurate diagnosis of patients with osteomyelitis including those with complicated diabetic foot disease.
Some people argue that PET/MRI will substitute PET/ CT for assessing the therapeutic success of treatments for chronic diseases, which requires repeated whole-body assessment of the extent of the disease, relapse, complications and concomitant diseases [20].

PET/MRI in cardiology Finally, cardiac applications have started to become the focus of attention of PET/MR adopters [27]. Historically, cardiac imaging has been a domain of research where one imaging technique would be replaced by another depending on the preferences and loyalties of the cardiac imaging specialists. However, Nekolla and co-workers discussed a few scenarios where combined PET/MR cardiac imaging may establish a new stage of cardiac diagnosis [27]. Combining PET with cardiac MRI and whole-body MR angiography may enable detection and differentiation of vulnerable plaques. The combination of late-enhancement MRI and $\left[{ }^{18} \mathrm{~F}\right]-\mathrm{FDG}$ uptake within a single imaging examination may expand the use of cardiac imaging. Initial studies combining MR spectroscopy with PET have already been performed on isolated perfused rat hearts, but may also enhance cardiac PET/MR studies involving cardiac stress simultaneously assessed with PET and MRI. Dual functional studies correlating the same parameters (e.g. perfusion in PET with radioactive water or ammonia and in MRI using arterial spin labelling or MRI contrast agents) can help to crosscorrelate and validate different acquisition techniques. PET tracer uptake, or PET perfusion, can be correlated with the MRI BOLD effect. Because of the large number of potential PET probes and the various functional imaging capabilities of MRI, the number of possible combinations for molecular imaging readouts is virtually unlimited. Simultaneously acquired PET and MRI data will allow accurate motion correction, particularly in cardiology, but also in the accurate detection of lesions in the abdomen or thorax [11].

\section{Methodological challenges}

Whole-body PET/MRI will become a key technological development in medical imaging technologies. Thus, prototype testing and validation studies today must be aimed at demonstrating reproducible imaging results with PET/MRI first. This entails accurate quantification, which, given the challenges of MR-based attenuation correction [11], is still not resolved. In this regard, ultra-short echo time (UTE) pulse sequences are being considered as part of an integrated PET/MRI examination in order to generate a signal from bone and, thus, provide means of better segmenting bone from non-bone tissues during the course of MR-AC. However, UTE sequences are known to be 
somewhat lengthy and, therefore, their adoption may be restricted by the overall duration of the study [28].

Clinically validated MR-AC methods must address adequate transformation of MRI pixel value information into appropriate PET attenuation values. In addition, MR image distortions must be detected, traced and, if possible, corrected during MR-AC. Such distortions include, for example, truncation and fold-in effects. Further, the presence of MR surface coils and positioning aids must be accounted for, both contributing to overall attenuation of the emission signal [15, 29-31].

In addition, cross-talk effects between MR gradients and PET electronics must be assessed under clinical imaging conditions. Finally, adequate workflow protocols must be designed and tested for a variety of clinical indications [23, 32, 33].

The question of sequential (Fig. 2a, b) or simultaneous (Fig. 2c) PET/MRI is the subject of an ongoing debate. From a technical perspective, simultaneous imaging allows for a number of advanced data processing steps that are not possible in sequential PET/MRI (and PET/CT imaging). This includes motion correction for involuntary patient motion and any subsequent quantification that may be biased from patient motion during the examination. To correct for patient motion, special MRI sequences can be applied by either one-dimensional navigator images or in two to three dimensions to detect the motion of the subject. Ideally, these protocols should be combined with the MRI sequence already running to provide motion information about the subject in intervals as short as $1 \mathrm{~s}$.

The overall advantage of truly simultaneous PET/MRI is that the same subject undergoes imaging at the same time with identical environmental parameters and stimuli. It is likely that such functional studies will further push the limits of basic biological research and will open new realms for studying biology in vivo.

Interestingly, there is potentially an immediate benefit for PET/CT from the ongoing development of PET/MRI. Studies by Kolb and co-workers have shown the large potential for novel types of APD [Geiger-APD (G-APD)] as light sensors for novel PET detectors. They can be operated with simpler electronics than those needed for APDs that are operated in linear mode; neither low-noise and charge-sensitive preamplifiers nor elaborate shielding is required. Further advantages of G-APDs over PMTs include their compactness, low operation voltages and insensitivity to strong magnetic fields [34]. It could be argued that G-APD-based detector designs, originally developed for PET/MRI, may eventually replace the PMT-based detectors in PET/CT systems and further stimulate the search for a common detector for both $\mathrm{CT}$ and PET [35].

\section{Small animal imaging systems}

Over the past decade we have witnessed a breathtaking increase in applications of molecular imaging instrumentation. Non-invasive, small-animal imaging, in particular, has excelled in catalysing molecular research and supporting translational research [36, 37]. Similar to human imaging, small-animal imaging systems were proposed to combine nuclear medicine technology with $\mathrm{CT}$ or MRI, thus providing co-registered functional and anatomical information, and to expand on the spatial coverage and sensitivity [38]. Figure 5 summarises a selection of dual- and tripletechnique small animal imaging systems available today.

However, the potential of small-animal imaging goes beyond detecting anatomical details or abnormal changes in morphology using high-resolution CT or MRI, and it extends towards revealing complex biochemical pathways or quantitative measurements of receptor, transporter or gene expression [39]. Functional imaging applications rely on methodologies like PET, SPECT or optical imaging (OI), providing excellent sensitivity to track biomolecules labelled with a radioactive isotope- or light-emitting marker. Nonetheless, it is not only the optical or nuclear methods that are able to provide functional information; fMRI and MRS have evolved to become powerful tools for detecting changes in blood flow, tissue oxygenation or concentrations of endogenous molecules such as lactate, choline or $\mathrm{N}$-acetyl-aspartate.

A dual-technique imaging combination for pre-clinical applications that has received a comparatively large amount of attention is PET/MRI. Interestingly, pre-clinical PET/MRI developments preceded clinical developments for a combination of PET and MRI [8], unlike PET/CT or SPECT/CT. Small-animal PET/MRI offers a number of advantages for pre-clinical studies [40], starting from significantly reduced exposure of the animal, thus paving the way for multiple repeat studies, the complementary acquisition of anatomical and multi-parametric image information through the use of MRI and much increased soft tissue contrast, making it easier to assess metabolic disease patterns in live animals.

Judenhofer and co-workers have demonstrated the feasibility of simultaneous small animal PET/MRI [41], and perhaps these types of dual-technique imaging systems will soon replace pre-clinical PET or PET/CT in dedicated small animal research laboratories. Further reasoning is provided by Wagenaar et al. [42]: "Simultaneous imaging is probably more important in the animal imaging domain than in clinical imaging. ... small-animal volumes are ten- to 1,000-times smaller, while heart ... and respiratory rate ... are up to ten-times higher. This means that a biological process that might develop in minutes to hours in humans can be over in seconds for a mouse ...". 

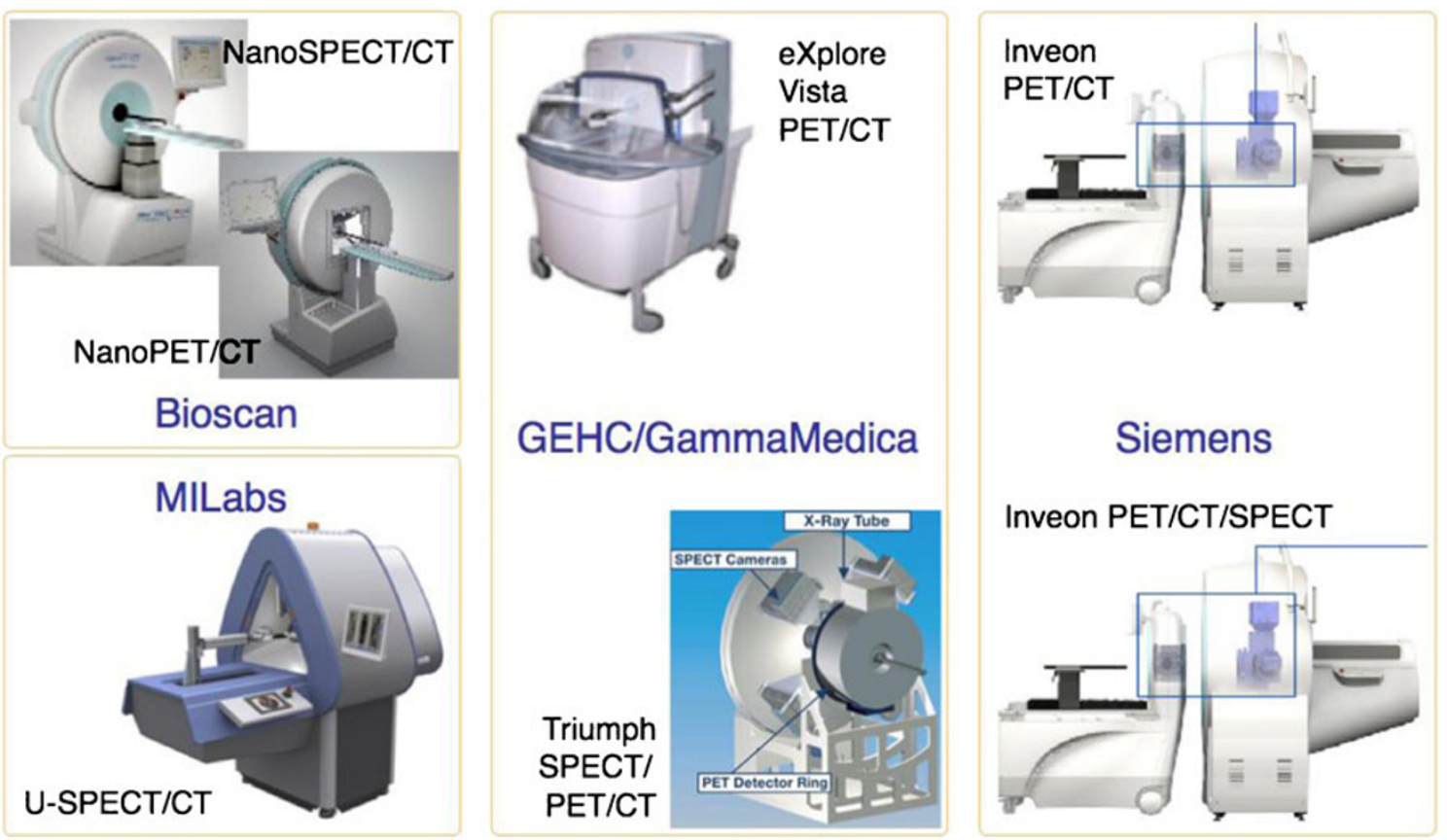

Fig. 5 Different design concepts for dual- and triple-technique imaging systems for pre-clinical applications. In general, system designs are similar to clinical dual-technique imaging systems even for the docking triple-technique system shown in the right panel

Small animal SPECT/CT has also been rather widely adopted, making use of the wide range of radiopharmaceuticals that can be produced independently of a cyclotron.

\section{Other imaging combinations}

Multi-modality imaging with PET/CT and SPECT/CT has become commonplace in clinical practice and in preclinical and basic biomedical research. But clinical multimodality imaging is not only limited to PET/CT, SPECT/ $\mathrm{CT}$ and PET/MRI, other imaging systems are currently in the design or exploratory phase (Fig. 6). The focus is generally on application-specific tasks such as imaging of breast and prostate. Examples are discussed briefly by Townsend [43] and include a combination of scintigraphy and mammography to reduce the false-positive rates from standard mammography, of three-dimensional (3D) CT breast imaging with SPECT or PET.

Recent advances in dedicated breast $\mathrm{CT}$ technology suggest that 3D mammograms are now possible, with no more radiation dose than from a two-view mammogram [44]. First studies indicate that the addition of intravenous contrast medium improved detection even further, and tumours that had not been seen with conventional mammography became visible. Some believe that by combining positron emission mammography (PEM) with dedicated $\mathrm{CT}$, or even dynamic contrast-enhanced (DCE) MRI, it should soon be possible to detect tumours as small as $1 \mathrm{~mm}$. The combination of ultrasound with other imaging techniques, such as conventional mammography and PET, has also gained increased attention from clinical researchers.

As some clinical multi-modality instrumentation originates from the pre-clinical domain, it is worth noting that there is commercial development of at least one SPECT/ MR device for small animal imaging [42, 45]. If a demand exists, this may eventually lead to a clinical SPECT/MR design.

Presently, the combination of PET or SPECT with MRI is an area of active prototyping, while the feasibility of other, perhaps less obvious combinations, including CT/MRI and PET/optical are also being studied [46]. In addition to the integration of the instrumentation, there are parallel developments in synthesising imaging agents that can be viewed by multiple imaging techniques [46].

\section{Other factors}

The future of hybrid imaging does not depend solely on the talents of system engineers and the drive of clinicians to make diagnosis more accurate through the adoption of more and more accurate imaging techniques. As the complexity of non-invasive diagnostic tools increases, so does the need for properly trained imaging experts.

Today, a decade after the first introduction of PET/CT, which originated from ideas raised in the realms of nuclear medicine, we see a large portion of PET/CT being employed merely as PET in combination with low-dose 

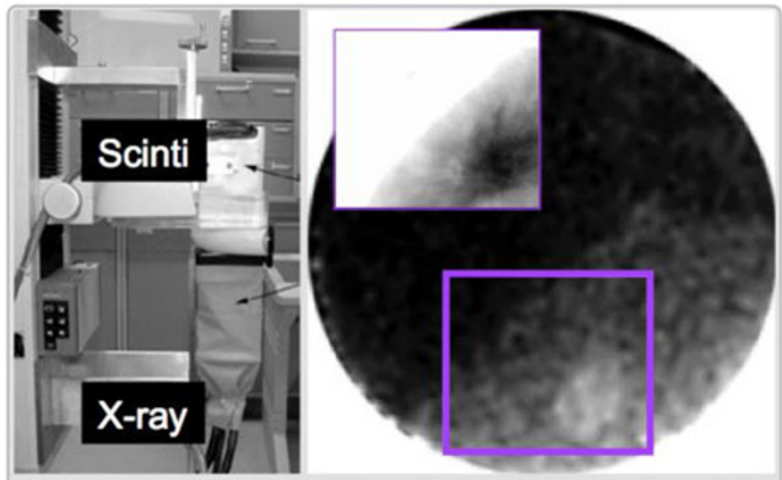

A

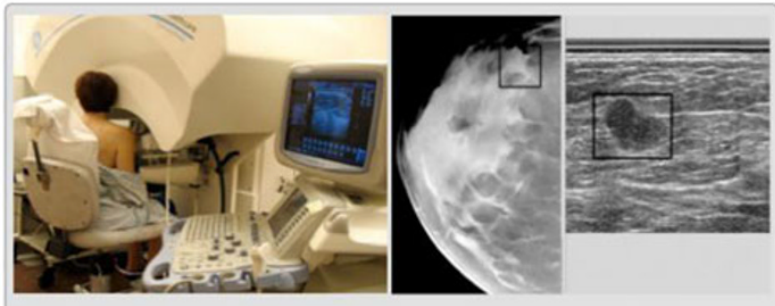

B
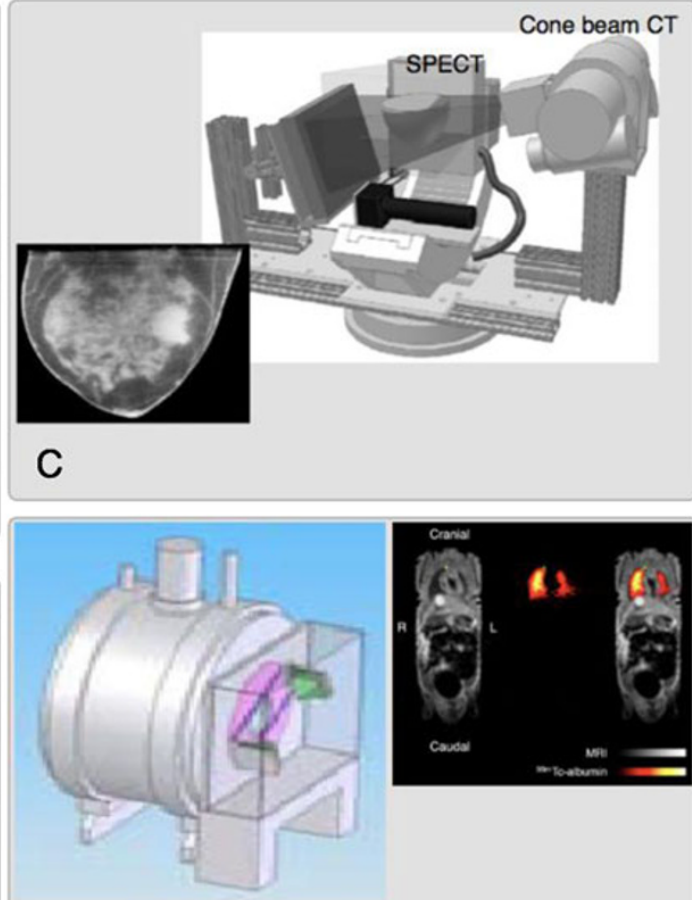

D

Fig. 6 Alternative combinations of imaging techniques in prototype designs and research testing: (a) scintimmamography imaging [58], (b) combined X-ray/ultrasound imaging [59], (c) mammotomography [60] and (d) SPECT/MRI [42]

CT to provide some anatomical background information [53]. This illustrates an important aspect of today's PET/ $\mathrm{CT}$, in that it is not always considered and utilised as a new imaging technique, which is partly related to interdisciplinary preferences and the lack of training. Therefore, joint efforts are promoted by the radiology and nuclear medicine imaging associations to provide sufficient training for combined imaging to young radiology professionals, making it very clear that "EANM and ESR recognise [that it] is important to provide adequate and appropriate training in the two disciplines in order to offer a proper service to the patient using hybrid systems. ..." [47].

However, adequate training alone is not sufficient to promote and adopt, where applicable, new hybrid imaging technology. Concessions have to be made for the vastly increased amount of data arising both from increased patient throughput as well as from the wealth of imaging information from a combined examination. The latter holds true in particular for PET/MRI examinations. It is assumed that there will be an $140 \%$ increase in imaging examinations by 2020 , and any advance in imaging technology must be matched by adequate advances in image assessment, which may support the use of computer-assisted image review tools. First approaches were tested in PET/CT with limited success [48].

Further, the adoption of new dual imaging techniques should be paired with the introduction of imaging guidelines [49-52]. A first evaluation of the adherence to PET/
CT guidelines has revealed surprisingly large deviations from guideline recommendations [53], which are related to deviations among guideline recommendations themselves and the lack of interest and knowledge in adopting standardised imaging protocols.

Finally, it is easily observed that reimbursement rates for dual imaging techniques differ widely internationally, even among industrialised countries [54]. Duplication of procedures and over-use of high-end procedures in situations where they add little clinical value has driven up technology spending [55]. While enthusiasm for new technologies grows quickly the adoption and reimbursement of these technologies in the future may be restricted and decided upon more carefully after sufficient technology assessment [56] or costbenefit calculations [57].

\section{The future of hybrid imaging: personal perspective}

Multi-modality imaging instrumentation has evolved dramatically during the past decade. Looking back to the year 2000, it is doubtful that one could have predicted the rapid clinical and commercial adoption of PET/CT, or the successful combination of SPECT with high-performance $\mathrm{CT}$, or the steadily increasing clinical interest in combining MRI with PET. PET/CT is now well established in the management of oncology patients, and the future will 
undoubtedly include continuing incremental advances in CT and PET instrumentation. A major contribution is, however, expected from the development and clinical introduction of new PET imaging tracers. These biomarkers will likely not replace FDG as a first-line imaging approach, but instead offer increased specificity and sensitivity in specific diseases and improved monitoring of therapy response; the choice of biomarker will be guided by personalised assessment of disease that includes genetic factors.

SPECT, and more recently SPECT/CT, is well established in the clinic, with an extensive range of labelled pharmaceuticals and the future is likely to involve detector developments in specific areas such as cardiology, and more quantitative methodology. Despite the increased cost of incorporating $\mathrm{CT}$, physicians will likely prefer to read SPECT with CT rather than SPECT without CT-the CT very much removes the "unclear" from the study.

In these times of greater economic hardship and increasing radiation awareness, any predictions for the future must take into consideration both cost-effectiveness and radiation dose. The impressive advances in imaging technology of the past decade came at a cost, but at what point do these advances becomes cost-effective? Wholebody PET examinations that took $1 \mathrm{~h}$ at the start of the last decade now take $5 \mathrm{~min}$ on PET/CT; the actual imaging takes only a fraction of the time needed for patient preparation and positioning or reporting the study.

The commendable drive to reduce radiation exposure to patients has turned attention to the combination of PET with MRI, a combination that represents substantial technical challenges beyond those of PET/CT. While these challenges have been overcome to a greater extent in the pre-clinical arena, not surprisingly combined PET/MRI is now eagerly awaited in the clinic. Indeed, the pre-clinical PET/MRI work can now be seen as an incubator for clinical design. So, will the coming decade witness the replacement of PET/CT by PET/MRI? Some believe it will, just as in the 1980s there were those who predicted that MRI would replace CT within 5 years. Of course that never happened, as both techniques have strengths and weaknesses and they have each found their niche in the medical imaging armamentarium. The same is likely true of PET/CT and PET/MRI - the technical challenges will be solved and simultaneous acquisition of MRI and PET will undoubtedly open new doors in clinical research and eventually also in the clinic.

The radiation dose to the patient incurred by PET, SPECT and CT is clearly an issue. Although the ALARA (as low as reasonably achievable) principle is sound advice, there are clearly groups of cancer-sufferers such as those in children and young adults where the probability of inducing a second, radiation-associated cancer exceeds the benefits that can be accrued from the study. Different imaging strategies should then be adopted, such as MRI, optical imaging or ultrasound. The next decade is likely to see nuclear imaging devices of greater sensitivity that can operate with even lower doses of injected activity, and more effective use made of the radiation incurred with multi-slice CT systems. As long as diseases such as cancer and dementia remain primarily diseases of the elderly, the benefits of nuclear and X-ray imaging will largely outweigh the risks.

Acknowledgements We are indebted to Dale Bailey (Sydney), Andreas Bockisch (Essen), Claude Comtat (CEA, Orsay), Bernd Pichler (Tübingen), York Hämisch (Bioscan), Matthias Hofmann (Tübingen), Ora Israel (Haifa), Antonis Kalemis (Philips London), Armin Kolb (Tübingen), Paul E. Kinahan (Seattle), Thomas Krause (Bern), Roger Lecomte (Sherbrooke), Marcus Lonsdale (Copenhagen), Bernd Schweizer (Philips Research, Aachen), Rainer Veigel (Philips, Zurich) for helpful discussions, advice and support materials.

Conflicts of interest T.B. is president and founder of Switzerlandbased cmi-experts $\mathrm{GmbH}$.

D.T. acts as scientific advisor to cmi-experts of Zurich, Switzerland and RefleXion Medical of Stanford, CA, USA. He received royalty payments from Siemens Healthcare related to the invention of PET/CT.

J.C. is co-founder of Momentum Biosciences and Sofie Biosciences, Los Angeles, CA, USA and serves as an advisor to cmi-experts $\mathrm{GmbH}$, Switzerland.

L.S.F. is an associate of ZRN Grevenbroich and Dormagen, serves as an advisor to cmi-experts of Zurich, Switzerland and has received speaker fees from Siemens, Philips and Genzyme.

Open Access This article is distributed under the terms of the Creative Commons Attribution Noncommercial License which permits any noncommercial use, distribution, and reproduction in any medium, provided the original author(s) and source are credited.

\section{References}

1. Padhani A, Miles K (2010) Multiparametric imaging of tumor response to therapy. Radiology 256(2):348-364

2. Hammer B, Christensen N, Heil BG (1994) Use of a magnetic field to increase the spatial resolution of positron emission tomography. Med Phys 21:1917-1920

3. Christensen $\mathrm{N}$ et al (1995) Positron emission tomography within a magnetic field using photomultiplier tubes and lightguides. Phys Med Biol 40:691-697

4. Shao Y et al (1997) Development of a PET detector system compatible with MRI/NMR systems. IEEE Trans Nucl Sci 44 (3): 1167-1171

5. Slates R et al (1999) Design of a small animal MR compatible PET scanner. IEEE Trans Nucl Sci 46:565-570

6. Pichler B et al (2006) Performance test of an LSO-APD detector in a 7-T MRI scanner for simultaneous PET/MRI. J Nucl Med 47 (4):639-647 
7. Judenhofer MS et al (2007) PET/MR images acquired with a compact MR-compatible PET detector in a 7-T magnet. Radiology 244(3):807-814

8. Wehrl $\mathrm{H}$ et al (2009) Pre-clinical PET/MR: technological advances and new perspectives in biomedical research. Eur J Nucl Med Mol Imaging 36(Suppl 1):S56-S68

9. Schmand $M$ et al (2007) BrainPET: first human tomograph for simultaneous (functional) PET and MR imaging. J Nucl Med 48 (6): $45 \mathrm{P}$

10. Von Schulthess G, Burger C (2010) Integrating imaging modalities: what makes sense from a workflow perspective? Eur J Nucl Med Mol Imaging 37(5):980-990

11. Hofmann M et al (2009) Towards quantitative PET/MRI: a review of MR-based attenuation correction techniques. Eur J Nucl Med Mol Imaging 36(Suppl 1):S93-S103

12. Zaidi H (2007) Is MR-guided attenuation correction a viable option for dual-modality PET/MR imaging? Radiology 244 (3):639-642

13. Beyer $T$ et al (2008) MR-based attenuation correction for torsoPET/MR imaging: pitfalls in mapping MR to CT data. Eur J Nucl Med Mol Imaging 35(6):1142-1146

14. Hofmann $M$ et al (2008) MRI-based attenuation correction for PET/MRI: a novel approach combining pattern recognition and atlas registration. J Nucl Med 49(11):1875-1883

15. Mantlik F et al (2011) The effect of patient positioning aids on PET quantification in PET/MR imaging. Eur J Nucl Med Mol Imaging. doi:10.1007/s00259-010-1721-9

16. Hofmann $M$ et al (2006) A machine learning approach for determining the PET attenuation map from magnetic resonance images. In: IEEE Nuclear Science Symposium and Medical Imaging Conference, San Diego

17. Brix $G$ et al (2005) Radiation exposure of patients undergoing whole-body dual-modality FDG-PET/CT examinations. J Nucl Med 46(4):608-613

18. Huang B, Law M, Khong P (2009) Whole-body PET/CT scanning: estimation of radiation dose and cancer risk. Radiology 251(1):166-174

19. Brix G et al (2009) Risks and safety aspects related to PET/MR examinations. Eur J Nucl Med Mol Imaging 36(Suppl 1):S131-S138

20. Pichler B et al (2011) PET/MRI: paving the way for the next generation of clinical multimodality imaging applications. J Nucl Med. doi:10.2967/jnumed.109.061853

21. Beyer T, Pichler B (2009) A decade of combined imaging: from a PET attached to a CT to a PET inside an MR. Eur J Nucl Med Mol Imaging 36(Suppl 1):S1-S2

22. Bisdas S et al (2009) Switching on the lights for real-time multimodality tumor neuroimaging: the integrated positron-emission tomography/MR imaging system. AJNR Am J Neuroradiol 31 (4):610-614

23. Boss A et al (2010) Hybrid PET/MRI of intracranial masses: initial experiences and comparison to PET/CT. J Nucl Med 51 (8):1198-1205

24. Heiss W (2009) The potential of PET/MR for brain imaging. Eur J Nucl Med Mol Imaging 36(Suppl 1):S105-S112

25. Antoch $G$ et al (2003) Whole-body dual-modality PET/CT and whole-body MRI for tumor staging in oncology. JAMA 290 (24):3199-3206

26. Antoch G, Bockisch A (2009) Combined PET/MRI: a new dimension in whole-body oncology imaging? Eur J Nucl Med 36(Suppl 1):S113-S120

27. Nekolla S, Martinez-Moeller A, Saraste A (2009) PET and MRI in cardiac imaging: from validation studies to integrated applications. Eur J Nucl Med 36(Suppl 1):S121-S130

28. Keereman V et al (2010) MRI-based attenuation correction for PET/MRI using ultra-short echo time sequences. J Nucl Med 51 (5):812-818
29. Delso $G$ et al (2010) Evaluation of the attenuation properties of MR equipment for its use in a whole-body PET/MR scanner. Phys Med Biol 55(15):4361-1374

30. Mantlik F et al (2010) The effect of positioning aids on PET quantification following MR-based attenuation correction (AC) in PET/MR imaging. J Nucl Med 51(Suppl 2):278P

31. Beyer $T$ et al (2010) The effect of MR radiofrequency coils on PET quantification in whole-body PET/MR. Eur J Nucl Med Mol Imaging 37(Suppl 2):S220

32. Schlemmer $\mathrm{H}$ et al (2008) Simultaneous PET/MRimaging of the human brain: feasibility study. Radiology 248(3):10281035

33. Ratib O et al (2010) Whole body PET-MRI scanner: first experience in oncology. J Nucl Med 51(Suppl 2):165

34. Kolb A et al (2010) Evaluation of Geiger-mode APDs for PET block detector designs. Phys Med Biol 55(7):18151832

35. Lecomte R (2009) Novel detector technology for clinical PET. Eur J Nucl Med Mol Imaging 36(Suppl 1):S69-S85

36. Cherry SR (2001) Fundamentals of positron emission tomography and applications in preclinical drug development. J Clin Pharmacol 41(5):482-491

37. Jong MD, Maina T (2010) Of mice and humans: are they the same?-Implications in cancer translational research. J Nucl Med 51(4):501-504

38. Meikle $\mathrm{S}$ et al (2005) Small animal SPECT and its place in the matrix of molecular imaging technologies. Phys Med Biol 50(22): R45-R61

39. Gambhir SS et al (2000) Imaging transgene expression with radionuclide imaging technologies. Neoplasia 2(1-2):118 138

40. Pichler B et al (2008) Positron emission tomography/magnetic resonance imaging: the next generation of multimodality imaging? Semin Nucl Med 38(3):199-208

41. Judenhofer $M$ et al (2008) Simultaneous PET-MRI: a new approach for functional and morphological imaging. Nat Med 14(4):459-465

42. Wagenaar D et al (2006) Rationale for the combination of nuclear medicine with magnetic resonance for pre-clinical imaging. Technol Cancer Res Treat 5(4):343-350

43. Townsend D (2008) Multimodality imaging of structure and function. Phys Med Biol 53(4):R1-R39

44. Frangioni J (2008) New technologies for human cancer imaging. J Clin Oncol 26(24):4012-4021

45. Hamamura M et al (2010) Development of an MR-compatible SPECT system (MRSPECT) for simultaneous data acquisition. Phys Med Biol 55:1563-1575

46. Cherry S (2009) Multimodality imaging: beyond PET/CT and SPECT/CT. Semin Nucl Med 39(5):348-353

47. Delaloye AB et al (2007) White paper of the European Association of Nuclear Medicine (EANM) and the European Society of Radiology (ESR) on multimodality imaging. Eur J Nucl Med Mol Imaging 34(8):1147-1151

48. Hahn S et al (2010) Computer-aided detection (CAD) and assessment of malignant lesions in the liver and lung using a novel PET/CT software tool: initial results. Rofo 183(2):243247

49. Delbeke D et al (2006) Procedure guideline for SPECT/CT imaging 1.0. J Nucl Med 47(7):1227-1234

50. Delbeke D et al (2006) Procedure guideline for tumor imaging with 18F-FDG PET/CT 1.0. J Nucl Med 47(5):885-895

51. Krause B et al (2007) FDG-PET/CT in oncology. German guideline. Nuklearmedizin 46(6):291-301

52. Boellaard R et al (2010) FDG PET and PET/CT: EANM procedure guidelines for tumour PET imaging: version 1.0. Eur J Nucl Med Mol Imaging 37(1):181-200 
53. Beyer T, Czernin J, Freudenberg L (2011) Variations in clinical PET/CT operations: results from an international survey among active PET/CT users. J Nucl Med 52(2):303310

54. Kotzerke J et al (2010) PET and diagnostic technology evaluation in a global clinical process. DGN's point of view. Nuklearmedizin 49(1):6-12

55. Goyen M, Debatin J (2009) Healthcare costs for new technologies. Eur J Nucl Med Mol Imaging 36(Suppl 1):S139-S143

56. Eisenberg J (1999) Ten lessons for evidence-based technology assessment. JAMA 282(19):1865-1869
57. Buck A et al (2010) Economic evaluation of PET and PET/CT in oncology: evidence and methodologic approaches. J Nucl Med Technol 38(1):6-17

58. Williams $M$ et al (2002) Combined structural and functional imaging of the breast. Technol Cancer Res Treat 1(1):39-42

59. Goodsitt M, Chan H, Hadjiiski L (2000) Stereomammography: evaluation of depth perception using a virtual 3D cursor. Med Phys 27(6):1305-1310

60. Madhav P et al (2009) Evaluation of tilted cone-beam CT orbits in the development of a dedicated hybrid mammotomograph. Phys Med Biol 54(12):3659-3676 